\title{
Coordinating different representations in the hippocampus
}

Eduard Kelemen ${ }^{\mathrm{a}}$ and André A. Fenton ${ }^{\mathrm{b}} *$

${ }^{a}$ Institute of Medical Psychology and Behavioral Neurobiology, University of Tübingen, Tübingen, Germany; German Center for Diabetes Research (DZD), Institute for Diabetes Research and Metabolic Diseases of the Helmholtz Center Munich at the University of Tübingen (IDM), Tübingen, Germany; National Institute of Mental Health, Klecany, Prague-East, Czech Republic; and Institute of Physiology, Academy of Sciences of the Czech Republic, Prague, Czech Republic

${ }^{\mathrm{b}}$ Center for Neural Science, New York University, New York and Department of Physiology and Pharmacology, Robert F. Furchgott Center for Neural and Behavioral Science, SUNY Downstate Medical Center, New York

*Corresponding author:

André A. Fenton

Center for Neural Science, New York

University, 4 Washington Place (Room 980), New York, NY 10003-6621.

E-mail: afenton@nyu.edu

(C) 2015. This manuscript version is made available under the Elsevier user license http://www.elsevier.com/open-access/userlicense/1.0/ 


\begin{abstract}
The processes that organize different thoughts and memories, allowing the separation of currently relevant and irrelevant information, are collectively known as cognitive control. The neuronal mechanisms of these processes can be investigated by place cell ensemble recordings during behaviors and environmental manipulations that present cognitive control challenges to selectively represent one of multiple possible alternative estimates of location. We review place cell studies that investigate responses to manipulations that dissociate the environment into two or more spatial frames of locations, often times to test notions of pattern separation. Manipulations, such as continuously rotating the recording chamber reveal that the ensemble discharge in hippocampus self-organizes into multiple, transiently-organized representations of space, each defined by the subset of coactive cells. Ensemble discharge in the hippocampus alternates between separate representations of frame-specific positions on timescales from $25 \mathrm{~ms}$ to several seconds. The dynamic, functional grouping of discharge into transiently co-active subsets of cells is predicted by the animal's changing behavioral needs. In addition to identifying neural correlates of cognitive control in hippocampus, these observations demonstrate that the separation of neuronal activity into distinctive representations depends on ongoing cognitive demands and that what can appear as noise, deviations from receptive field tuning, can substantially be the result of these internal knowledge-guided fluctuations. These findings inspire a new perspective that should be taken into account when investigating pattern separation - a perspective that emphasizes changes in hippocampal neural discharge that are happening on a short timescale and does not assume that patterns of neural discharge are steady and stationary across the several minutes of the recordings.
\end{abstract}

\title{
Keywords
}

Dynamic functional grouping, multiple representations, cognitive control, hippocampus, overdispersion 
1. Introduction

To succeed in life people and other animals must not only acquire vast amounts of information about the world, but they must also be able to distinguish the information that is worth using and learning, from what is irrelevant. To be adaptive they must selectively use the appropriate information in appropriate situations. Mammals must react quickly to the changing environment and often it is necessary to multitask. The processes behind the ability to select and use appropriate information at proper situations, specifically the processes of goal-directed action selection, response activation and inhibition, performance monitoring, and reward-based learning, are collectively called cognitive control (Posner and Snyder, 1975; Ridderinkhof et al., 2004). While, in the past a lot of abundantly rewarded attention has been paid by the neuroscience community to the neural processes underlying single neural representations and the formation and storage of single memories, the neuronal processes that organize and manage multiple neural representations and memories are becoming an area of increasing research interest, in part because cognitive control inability is a prominent symptom in diverse forms of mental illness (Phillips and Silverstein, 2003; Uhlhaas and Singer, 2006, 2007; Carter and Barch, 2007; Lesh et al., 2011). Subtle manipulations and monitoring of hippocampus function has implicated a role of hippocampus in cognitive control functions (Wesierska et al., 2005; Olypher et al., 2006) and importantly, study of hippocampal physiology offers a neural system optimized for investigating the basic neural information processing mechanisms that may underlie cognitive control. Here we review recent progress in this research effort to investigate the hippocampus role in cognitive control.

The neural computations of pattern separation (increasing the difference between two similar input representations) and pattern completion (decreasing the difference between similar input representations) (Marr, 1971; O'Reilly and McClelland, 1994) have been traditionally studied by making discrete environmental dissociations such as using a circle-to-square morphable box (Leutgeb et al., 2005a; Wills et al., 2005) or double rotation experiments (Tanila et al., 1997a; Knierim, 2002; Brown and Skaggs, 2002; Lee et al., 2004). Such studies are predicated on an assumption of representational stationarity, an assumption that we question. The standard analysis of firing fields assumes that the responses of cells will be qualitatively the same throughout the experience of the recording session in a stable experimental environment. This implies that a cell's characteristic firing is appropriately captured by the time-averaged response of the cell. However, we observed representational switching indicative of pattern separation but the process is dynamic and multistable, in violation of the stationary assumption. This multistability is observed in standard 
place cell recordings but becomes especially apparent during environmental dissociations that are continuous.

2. Early studies - assessing the organization of distinct hippocampal representations by manipulating independent sets of landmarks between experimental sessions

After the discovery of hippocampal place cells - principal neurons with a location-specific organization of discharge (O'Keefe and Dostrovsky, 1971) - an elegant method of constructing firing rate maps was used extensively to characterize place cell spatial discharge properties (Muller et al., 1987). A firing rate map is created by computing for each location that was visited, the average firing rate of the neuron. A firing rate map averages the activity of a cell over the entire recording session, which typically lasts several minutes. The tacit assumption is that the activity in each location is appropriately represented by the session-averaged firing rate. The method was immensely successful and was instrumental for discovering many fundamental properties of place cells, and to this day shapes the way experiments are planned and data are analyzed and interpreted. This way of analysis and data presentation is implicitly oblivious to processes that might be occurring on short time scales, relative to the several minutes it takes the animals to visit most locations in the environment. Partly due to the success of this method, hippocampal place cell activity was often implicitly considered to be a system that does not change spontaneously on a short timescale. It was assumed that the place cells respond to the location of the animal (or other task-related parameters) essentially the same way, at every moment of a recording session.

A number of studies investigated changes in place cell responses across subparts of experimental tasks, for example on inward and outward journeys on a radial arm maze (McNaughton et al., 1983), or during cue-sampling and goal approach in odor discrimination tasks (Eichenbaum et al., 1987), and distinct phases of matching-to-sample tasks (Hampson et al., 1993), as well as before and after moving a prominent visual cue (Sharp et al., 1995; see reviews Redish, 1999 and Muller, 1996) or during different subparts of spatial alternation tasks (Wood et al., 2000). Some studies reported changes in firing that occurred in response to changes in task within an environment (Markus et al., 1995), but the organization of these different firing patterns was rarely systematically studied on a the time scale of seconds, largely because of the emphasis on single cell firing fields as the unit of study. Notable exceptions are a set of investigations that pioneered studies of the representational dynamics of positional information in the discharge of place cell ensembles (Gothard et al., 1996a; Gothard et al., 1996b; Redish et al., 2000; Rosenzweig et al., 2003). Relatively few other studies 
reported changes in place cell firing within a session (Knierim et al., 1995; Tanila et al, 1997b; Knierim, 2002), but again, with a focus on the session-averaged properties of the phenomena, rather than the dynamics of the potential changes.

Early studies that aimed at the question of how multiple representations are processed within the hippocampus were designed, performed and interpreted in the analytical framework that was established by session-averaged firing rate maps. A number of these early studies used a "double rotation" experimental design (Figure 1A): To probe how the hippocampal representation reacts when two different interpretations of environmental landmarks are possible, the same set of place cells was compared in two recording sessions: in a standard session with two sets of landmarks present, and then again in a double rotation session with one set of landmarks rotated in one direction, and the other set of landmarks rotated in the opposite direction (Tanila et al., 1997a; Fenton et al., 2000a,b; Knierim 2002; Brown and Skaggs, 2002; Lee et al., 2004). The results somewhat varied depending on the details of a particular experiment, but they allow general conclusions. In the hands of Fenton et al. (2000a,b), who performed the smallest perturbation-like changes in the landmark composition, the place cell representation was distorted but remained cohesive, so that each cell's firing rate map responded to the position of both displaced landmarks. In most other experiments, the majority of CA1 place cells responded consistently, following one set of landmarks (Tanila et al., 1997a; Brown and Skaggs, 2002). But some of the CA1 cells responded independently of the majority (Tanila et al, 1997a, Shapiro et al., 1997) and a significant proportion followed the other (non-dominant) set of landmarks (Knierim, 2002, Figure 1B). A stronger tendency for a consistent response across the population of cells was observed in CA3 than in the CA1 hippocampal subfield (Lee et al., 2004).

The question of multiple representations in hippocampal discharge was investigated by Gothard et al. (1996a) using a different strategy. They used a spatial task in which a rat in an open field started a trial in a box, looked for food located relative to two local landmarks, and then returned to the box at the end of the trial. The positions of the box at the beginning of the trial, the positions of the box at the end of the trial, as well as the position of landmarks marking the reward location were changed from trial to trial. This allowed the authors to study place cell representations of the rat's position in different reference frames: relative to the box's variable start location, relative to the box's variable end location, relative to the variable reward location, as well as relative to the rat's position in the stationary room. The authors observed hippocampal place cells responding to the room location, as well as cells discharging in relationship to the start-box, end-box and reward locations. Similarly, Muller and Kubie (1987) and Rivard et al. (2004), observed cells responding to 
the rat's position relative to distal landmarks as well as cells responding in the vicinity of locally placed barriers. Overall these results show that in addition to the general tendency for a uniform across-cell response in CA1 ensembles of cells, some cells can also act independently of the majority to signal locations with respect to other independently moved salient or significant cues. All these studies share a feature that limits their interpretation - the cells' spatial responses were averaged over the entire session. Changes that potentially could have happened on a sub-session timescale were not considered and would have been hard to detect by the analysis of session-averaged place fields.

The dynamics of place cell representation changes within a recording session was first assessed by the Barnes and McNaughton laboratory (Gothard et al., 1996b; Redish et al., 2000; Gothard et al., 2001; Rosenzweig et al., 2003). Their approach was to create, within a single session, a conflict between the rat's self-motion positional information and positional information about the distal landmarks. In their typical experiment place cell discharge was recorded while a rat ran between two endpoints on a linear track. One end of the track stayed in a stable position in the room; the other end of the track was defined by a box that could be moved to vary the distance between the box and the stable end of the track on each trial (Figure 2). Place cell discharge was aligned with the box at the beginning of the journey, and with the end of the track at the end of the journey. Somewhere along the track the representation switched from being box aligned to being end-of-the-track aligned. The authors detected smooth transitions, especially if the conflict between the self-motion cues and distal cues was relatively small (Figure 2B), and also abrupt transitions, if the conflict was large (Figure $2 \mathrm{C}$ ). The precise time of transitions between the representations could be determined on individual laps (Redish et al., 2000; Gothard et al., 2001; Rosenzweig et al., 2003). Overall these results showed that by clever manipulation of the experimental situation a change between distinct place cell representations can be induced within a session. Can such changes between place cell representations occur spontaneously?

3. Spontaneous changes in hippocampal spatial representations within a single session overdispersion in place cell firing

The studies we have discussed thus far were performed under the tacit assumption that as long as the landmarks or other aspects of the task are not manipulated in an experiment, place cell activity remains the same throughout an experimental session, with some noise inherent to any biological system. This notion was challenged by the report that "place cell discharge is extremely variable 
during individual passes of the rat through the firing field" (Fenton and Muller, 1998). The analysis showed that even though the behavioral contingencies did not change in standard, stable and familiar environments, during different passes through place cell's firing field, the discharge of the cell varied more than would be predicted by the variability of a random process (Figure 3 ). This phenomenon was later called "overdispersion" (Olypher et al., 2002) and a statistical model indicated that the observed variability in place cell discharge could be expected if a cell's spatial firing was controlled by two distinct processes that alternate on a timescale of a few seconds (Olypher et al., 2002). Other groups independently pursued the idea of dynamic short timescale changes in hippocampal representations. Harris et al. (2003) showed that CA1 cells form temporal coalitions - from Hebb (1949) they borrowed the term cell assemblies for them - and uncovered evidence that the temporal coalitions are organized by ongoing gamma oscillations. Through these studies the notion that there were important, short timescale dynamics in hippocampal activity was making its way to the mainstream thinking about hippocampal function.

The initial studies of overdispersion suggested that place cell representations of space are switching between different distinct activity states every few seconds but it was not known whether the discharge of individual hippocampal cells varied independently or if the discharge variance was coordinated as if the cells were switching between global, coactivity-defined states. Could it be that hippocampal activity as a whole was toggling between different states, in the standard place cell recording conditions? Jackson and Redish (2007) and Fenton et al. (2010) provided compelling evidence that indeed the changes are coordinated across ensembles of cells as if multiple spatial maps were alternately present in hippocampal ensemble discharge, within a single task in the standard place cell recording paradigms. Switching between two representations was observed during foraging and maze running behaviors on linear track and cylindrical open field environments, as well as during a place preference navigation task. Jezek et al. (2011) provided further, powerful support to the notion that hippocampal ensemble activity can rapidly toggle between distinct states, corresponding to distinct spatial representations. They showed in recordings of the hippocampal CA3 subfield that in response to abrupt changes between two familiar visual cue sets, the CA3 ensemble discharge toggles between the two distinct representations and that this switching is organized on the time scale of single hippocampal theta oscillations, that control when the switches occur. These studies showed that not only single neurons, but hippocampal ensemble activity as a whole switches between two or possibly more states every second or two. These data demonstrate that hippocampal representations of location are multistable on the time scale of seconds, in addition to the long-time scale multistability that was described as remapping. To summarize, we borrow the title of Jackson and Redish (2007): “Network dynamics of hippocampal cell assemblies 
resemble multiple spatial maps within single tasks." The overdispersion findings were not initially embraced by the community, in large part because it was unknown what the behavioral significance of switching between multiple maps of the same fixed environment might be, especially given the switching happened every few seconds.

4. Behavioral significance of fast switching between multistable representations

It was hypothesized that overdispersion in place cell discharge is because the rat switches between locating itself in more than one distinctive spatial reference frame, in a process that might resemble the influence of attention on place cell firing (Fenton et al. 2010). In support of this hypothesis, overdispersion is reduced when the rats perform a place preference task that requires going to an unmarked goal to trigger the release of food to a random location, and foraging for the scattered food. Overdispersion was reduced even further, after rats learned to do the place preference task by selectively using only the distal subset of landmarks and ignoring the local cue subset. This was achieved by putting rats on a continuously, slowly rotating $(1 \mathrm{rpm})$ circular arena and reinforcing them to visit the goal location that was defined only by the stationary room landmarks because the rotating local positions did not define the goal. These observations confirmed that overdispersion is organized across the population of place cells, but they only suggested that the switching between discharge states reflects what information is being used to navigate.

The behavioral significance of the switching between multistable representations was studied further using an experimental paradigm that directly tested the rat's ability to use two types of concurrent spatial information (Figure 4A; Kelemen and Fenton, 2010; 2013). The rats had to multitask: they were placed on a continuously slowly rotating arena and had to avoid two shock zones. One shock zone was defined by room landmarks and remained stationary within the room. The other shock zone was defined relative to cues on the arena, such as scent marks made by the rat that rotated together with the arena. To avoid mild foot shock, the rat had to know where it was relative to the room landmarks, as well as relative to the arena landmarks. Both arena and room positions were represented in hippocampal CA1 discharge that was recorded during the task. An information theoretic measure was used to estimate separately the amount of information about position in the room and arena spatial frames from the ensemble discharge during each $120 \mathrm{~ms}$ period. This estimate showed that periods of predominant information about arena position alternated with periods of predominant information about room position. This dynamic functional grouping of neural activity into transient, same-function subgroups of coactive cells, showed that place cell ensemble discharge alternates between signalling locations in specific spatial frames 
according to internal cognitive variables such as the cognitive control state. Furthermore, whether room or arena position was predominantly represented at a particular moment reflected the behavioral relevance of the two spatial frames at that moment. Specifically, the information about the current position in the room dominated ensemble activity when the animal was close to the stationary room shock zone, and information about the position on the rotating arena dominated when the animal was close to the rotating arena shock zone (Figure 4B,C). These studies provide direct evidence that place cell ensemble discharge is multistable, alternating between representing locations in distinct spatial frames according to moment-to-moment behavioral needs. While this view is rooted in the initial observations and analysis of overdispersion, it was only possible to assign and demonstrate the behavioral significance of the spiking variability, when the rat was performing a properly designed task.

We will now briefly consider these alternations in the internal representation of places in the environment from the perspective of overdispersion. Because an observer cannot typically know the moment-to-moment state of an information representation that fluctuates according to internal processes, those cognitively-essential fluctuations will appear as unexplained deviations from the verifiable expectations that are characterized by a tuning curve. In other words the deviations will appear as noise, despite its non-random statistical organization (Olypher et al., 2002). Here, we exploited the Kelemen and Fenton (2010) data set to illustrate this point by estimating overdispersion during the multitasking behavior in which the dynamic functional grouping of place cell ensembles was identified. Each ensemble spike-and-position time series was subdivided into the episodes when room information predominated in the CA1 ensemble, and the moments when arena information predominated (Fig. 5A). The firing rate deviation of each place cell's discharge during each frame-specific episode was computed as a z score according to the Poisson expectation from the session-averaged firing rate map (Fig. 3). The assertion that overdispersion reflects a hidden, attention-like cognitive variable like cognitive control predicts that the variance of the $z$ scores during only the room-preferring or only the arena-preferring episodes will be substantially reduced because the activity in each condition is from a more homogeneous cognitive state. Indeed, overdispersion estimated by the variance of spiking is typically about 5 for CA1 (Fenton and Muller, 1998; Jackson and Redish, 2007; Fenton et al., 2010) but it was only 2.40 in the room spatial frame during the room-preferring episodes and only 2.19 in the arena spatial frame during the arenapreferring episodes when we considered all episodes longer than 3 seconds. Three seconds was chosen to match the statistics of the duration of passes through firing fields that have been investigated to characterize overdispersion in prior work (Fenton and Muller, 1998; Fenton et al., 2010). These values were computed from any episode where the rat was detected in a location 
where the cell was active and thus the expected firing was non-zero, which is the minimum requirement for defining a z score. The variance was not significantly increased (arena-frame firing during arena-preferring episodes: $v a r=3.06$ and room-frame firing during room-preferring episodes: $\operatorname{var}=2.86$; Figure $5 \mathrm{~B}$ ) when the activity criterion was increased to require the expected activity during the episode to exceed the session-average rate, which selects for episodes when the rat passes through a substantial portion of the firing field (Fenton et al., 2010). To test that the reduced overdispersion was not due to the mere act of subdividing the data into two arbitrary classes of episodes, we recomputed the values for the arena-frame firing during the room-preferring episodes and for the room-frame firing during the arena-preferring episodes. Overdispersion was again approximately 5 (arena-frame firing during room-preferring episodes: var $=4.94$ and room-frame firing during arena-preferring episodes: var $=6.39$; Figure 5B). In strong support of interpreting overdispersion as the result of an internal, attention-like cognitive process such as cognitive control, the room-frame $\left(t_{1475}=3.33 ; p=0.0009\right)$ and arena-frame $\left(t_{1544}=2.57 ; p=0.01\right)$ variances were significantly smaller when they were computed within the appropriately corresponding framepreferring episodes.

The investigation of information about internal cognitive variables in place cell ensemble discharge was further expanded. In addition to organizing different representations of the current space, dynamic functional grouping also organized the retrieval of different memories (Kelemen and Fenton, 2013). The rats performed two different place avoidance tasks in the same physical environment. One task was performed with the arena stationary (not-rotating), and the other with the arena rotating. Well-trained rats performed the two tasks on the same day - one session on the rotating arena flanked by two sessions on the stationary area. Although both tasks were performed in the same physical space, the CA1 representation of the two tasks was different (i.e. remapped), similar to how hippocampal activity was reported to remap when distinct behavioral tasks were performed in the same physical environment (Markus et al., 1995). The key finding of this study with the two place avoidance tasks, was that during the rotating task, from time to time, activity characteristic of the stationary task appeared in CA1 ensemble discharge. The details of when these events occurred, as well as what the rat was doing and what positional information hippocampal discharge was representing, indicated that these dynamic functionally grouped patterns of coactivity are the neural manifestation of non-local, cross-episode memory retrieval events. These experiments extend the findings of Kelemen and Fenton (2010) and show that dynamic functional grouping can also organize representations of distinct behavioral episodes during active behaviors in the online, theta-dominated activity state of the hippocampus, not just in offline sharp-wave associated ripple states as has been described for replay of hippocampus place sequences (Gupta et 
al., 2010, Carr et al., 2011). Consider also another interesting example of the behavioral role of representation switching. CA1 place cell ensembles were recorded while rats performed a series of T-maze left/right discriminations. At the junctions of the left/right choices, when the rat must decide between making a left or right turn, CA1 place cell representations of the two alternative future trajectories were activated as coactive, functionally grouped discharge patterns. As if through vicarious trial-and-error, the rat considered the two options by imagining the consequences of each, before making the decision (Johnson and Redish, 2007). Dynamic functional grouping emerges as a potentially universal mechanism. It not only organizes distinct concurrently relevant streams of information, but it also organizes the reactivation of representations stored in memory, as well as the activation of information representations for evaluating future options.

\section{Timescale of ensemble representation switching}

A number of studies have investigated the timescale of switching between distinct hippocampal representations. The results on the transition times are remarkably consistent across studies from several laboratories. A statistical analysis and model of overdispersion concluded that during foraging in an open field, individual place cell representations alternate with a period of approximately one second (Olypher et al., 2002). Analysis of place cell ensembles during the same behavioral conditions also concluded that the average dwell-time in a state was about one second Fenton et al. (2010). These results are not far from the mean rates of switching of several hundred milliseconds that were observed by Jackson and Redish (2007) during similar behaviors. During twoframe place avoidance on a rotating arena, where separate processing of positions in the room and arena spatial frames was reinforced, the preference in ensemble activity for one or the other spatial frame also toggled at the timescale of hundreds of milliseconds Kelemen and Fenton (2010), whereas unreinforced switching between the representation of the current task and the crossepisode retrieval of the representation of the previously performed task happened on a slower timescale of about 7 seconds (Kelemen and Fenton, 2013). Harris et al. (2003) observed that dynamic functional grouping organized cells into coactive subgroups at the 25-ms timescale of gamma oscillations, such that same-function coactivity signalled the specific location of the rat during unreinforced foraging behavior. Gamma-scale organization was also observed during twoframe place avoidance on the rotating arena. Pairs of place cells that signalled position in the same spatial frame during 120 ms periods also tended to discharge within $25 \mathrm{~ms}$ of each other, the period of a gamma oscillation. In contrast, although cell pairs that discharge together in 120 ms rarely signalled positions in opposite spatial frames, when this discordant event happened, there 
was no gamma-scale coactivity preference for the spike timing. These studies suggest that multiple mechanisms organize place cell activity into coactive same-function subgroups to signal distinct qualities of location that include position, spatial frame, and behavioral episode. Although the specific mechanisms have not been fully clarified, they seem to involve the dynamic coordination of excitation and inhibition, operating on multiple timescales much shorter than the several minutes of the typical analysis of place fields (Buzsaki, 2010). Over averaging as is necessary to characterize place fields would certainly have made it difficult to recognize the varieties of dynamic functional grouping phenomena and perhaps impossible to discover the mechanisms.

6. Mechanism of dynamic functional grouping - hypothesis

The cornerstone of dynamic functional grouping hypothesis is the notion that the units of the network switch representations together as an ensemble rather than independently (Harris et al., 2003; Jackson and Redish, 2007; Fenton et al., 2010; Kelemen and Fenton 2010; Kelemen and Fenton, 2013; Jezek et al., 2011). This evidence favors the hypothesis that hippocampus is acting as an organized unit, rather than a composition of independent neurons to signal location (Fenton et al., 2008; Harris et al., 2003), which is likely similar for the neocortex, although individual cells can appear to be at either end of a continuum between substantial and limited independence of the overall population (Okun et al., 2015). Perhaps, the best elaborated model of ensemble organization of hippocampal activity is the attractor network model (Samsonovich and McNaughton 1997; Redish, 1999). The gist of the model has a group of mutually activated neurons - coding for a single location - activated and the global inhibition that prevents other neurons from firing. The model asserts that attractor properties of steady and stationary dynamics are built into the architecture of the hippocampal network. This can explain the tendency for only one representation to be active at a particular time. Switching between different representations is an elegant solution to the problem of how to activate representations that are each encoded by a different attractor state within a single network. Depending on the strength and time course of coordination between excitation and inhibition in such networks, under certain conditions these network dynamics allow for overlap between different representations, such as in case of spurious attractors (Knierim and Zhang, 2012).

While theta oscillations organize hippocampal discharge in general, they also play an important role in organizing the activation of different information representations in the hippocampal discharge (Jezek et al. 2011). This was shown in the CA3 region of the hippocampus, where it is thought that a higher number of mutual connections between the principal cells provides for an attractor architecture and dynamics. Theta oscillations organize hippocampal neuronal activity into distinct 
phases with distinct functions (Hasselmo, 2005). During the trough of pyramidal layer theta, pyramidal neurons are active, stimulate each other, and process information. During the peak of pyramidal layer theta, neuronal activity is relatively suppressed, and input from entorhinal cortex is increased providing an opportunity to switch to a new representation if the conditions favor it. The data on timing of the switching, which show that it occurs on the order of hundreds of milliseconds to seconds, indicate that representational switching occurs after several theta cycles, which implies there are additional processes in addition to theta oscillations that organize switching (Figure 6).

The idea that an attractor state is being established within each theta cycle predicts that the stability and perhaps other qualities varies with the phase of theta. Consistent with findings from Jezek et al. (2011), in the theta phase when the activity of principal neurons starts to increase, the stationary state may not yet have settled and different potential representations may be present and competing for dominance in ensemble discharge. This competition should have been resolved towards the end of the theta cycle and at this time a single representation should be present in ensemble discharge.

How are the activated representations selected from the several that are possible? According to the attractor notions, it would appear that a key to this process is the comparison and matching of the currently active representations, with the currently present sensory input and behavioral contingencies. In this context, the difference between the functional properties of different hippocampal subregions may be of some consequence. In the case of ambiguous sensory input such as an environment with similar sensory conditions at multiple locations, the CA3 network appears to readily activate a pre-learned representation (Lee et al., 2004; Vazdarjanova and Guzowski, 2004; Leutgeb et al., 2004; Guzowski et al., 2004; Wilson et al., 2005) that best matches the current stimuli and behavior. The CA3 properties predispose it to finding the best interpretation of the incoming signals. In the same ambiguous conditions, CA1 activity is driven by pre-learned representations to a lesser extent and better than CA3, CA1 activity reflects the specifics of the current sensory input. The CA1 network receives inputs from CA3, as well as from layer 3 of the entorhinal cortex (EC3), and so it can compare the EC3 version of the input comprised of grid, border, and directional cells with its CA3 interpretation comprised of place cells. CA1 is in a unique position to detect discordance between the model of the world offered by CA3 and the more raw information from EC3. On the mechanistic level, in the case when consistent information is present in the model and the raw signals, inputs from CA3 and EC3 would converge on the same strongly activated CA1 pyramidal cells (Figure 7A). In the case of discordance between the model and the raw data, EC3 input and CA3 input would impinge on different activated CA1 pyramidal cells (Figure 7B). The result of this 
comparison is transmitted from CA1 back to EC where it affects and can alter activity (Bonnevie et al., 2013) and could stimulate a switch in representations. As presented in Figure 7, only the feedforward excitatory connections are crucial and the focus is on feed forward information flow.

Discordant versus concordant inputs to CA1 appear to be reflected in single unit activity as well as in the oscillatory synaptic population activity recorded as oscillations in the local field potential. While local field potential theta oscillations provide surprisingly high-fidelity spatial information about the animal's position and the underlying hippocampal activity (Agarwal et al., 2014), gamma oscillations primarily reflect synchronous population inhibitory currents (Buzsaki et al., 2012). EC3 and CA3 inputs to CA1 are associated with different gamma oscillations with dendritically localized input currents and distinctive inhibition in dendritic and perisomatic regions of CA1 (Lasztoczi and Klausberger, 2014, Schomburg et al., 2014). Thus, as depicted in Figure 7, the feed-forward excitatory picture is missing this dynamic, inhibitory component. The same excitatory afferents on the CA1 pyramidal cells, also contact CA1 inhibitory cell subtypes with dendrites in a particular stratum radiatum or lacunosum-moleculare dendritic input compartment. This architecture creates competition between the excitatory and inhibitory consequences of afferent activity, with inhibition at a given moment typically dominating most principal cells, except the minority that have sufficiently escaped inhibition to be biased towards responding to the excitation that is depicted in the Figure 7 schematics. A comprehensive and validated description of the inhibitory neuron types and their properties, necessary to implement such a competitive-network architecture is lacking, but clear progress is afoot (Royer et al., 2012).

\section{Implications and future directions}

The work reviewed here shows that the short timescale dynamics of hippocampal discharge is important and worth closer attention. By averaging the activity of neurons throughout an experimental session lasting several minutes, important information is lost. This may have implications in interpreting some of the earlier results in the research field, in particular when the dynamics of pattern separation and pattern completion processes have been considered as monostable and stationary. In light of dynamic functional grouping, these findings predict that in the early double rotation experiments (Tanila et al., 1997a; Knierim, 2002; Brown and Skaggs, 2002; Lee et al., 2004) the cells responding to room landmarks and arena landmarks were active at different times rather than in parallel. Knierim (2002) addressed this question by creating cross-correlations between the activity of pairs of cells with overlapping firing fields that rotated with different sets of 
landmarks. The analysis of 11 cell pairs showed no indication of cells being active at different times, within $10 \mathrm{~ms}$, although this is not typical of what we have observed during navigation, whether or not the arena is stationary (Kelemen and Fenton, 2012) or rotating (Kelemen and Fenton, 2010) or even when the rat is anesthetized (Olypher et al., 2006). In so-called morph experiments, which showed changes in firing rate maps as the environment was gradually changed between two extreme shapes - a circle and a square (Leutgeb et al., 2005a; Wills et al., 2005), the dynamic grouping findings predict that intermediate representations during the transitional states were the result of the two extreme representations being active at different times within the same session. In a similar vein, the phenomena of 'partial remapping' (Muller and Kubie, 1987; Muller, 1996) and 'rate remapping' (Leutgeb et al., 2005b) could be at least partially explained by different representations being activated at different times. Skaggs and McNaughton (1998) analysed whether partial remapping could be explained by such effects and reached inconclusive results. The dynamic grouping perspective predicts that transient changes related to pattern separation and pattern completion are occurring and on short, sub-second timescales, which is a perspective that will require further investigation.

Another open question is the control of initiating the switches between representations. In the conceptual model we described above, we discussed the option that the switching is initiated within the hippocampus, in the CA1 region. The switching could also be initiated upstream of the hippocampus. There is clear evidence that it might be initiated in frontal cortex and conveyed to CA1 via nucleus reuniens (Ito et al., 2015).

The organization of hippocampal discharge switching between different representations on short timescales may be intimately related to our notions about cognitive control. The dynamic grouping of activity into cell assemblies of coactive cells and the dynamic transitions between different activity patterns may comprise or at least resemble the neural mechanisms that implement goaldirected action selection, response activation and inhibition, performance monitoring, and rewardbased learning that are collectively called cognitive control (Ridderinkhof et al., 2004). Because impairments of cognitive control are a core cognitive symptom in schizophrenia, understanding the mechanisms of cognitive coordination may be crucial in uncovering pathophysiological mechanisms of the disease, and other forms of mental illness (reviewed in O'Reilly et al., 2014; Fenton, 2015).

In summary, a new way of looking at hippocampal activity is emerging and gaining experimental support. Recent findings show that hippocampal activity is changing between different representations on multiple timescales of tens and hundreds of milliseconds, and several seconds, hours (Manns et al., 2007; Mankin et al., 2012) and possibly even days (Ziv et al., 2013). This 
organization of neural population activity is such that the coactive subset of cells provide for a similar information coding function. This dynamic grouping of neural discharge appears to be internally generated, but also reflects ongoing and changing behavioral needs.

Acknowledgements: Supported by National Science Foundation grant IOS-1146822, National Institute of Mental Health grant R01MH084038, Deutsche Forschungsgemeinschaft (TR-SFB 654), German Federal Ministry of Education and Research (BMBF) grant to the German Center for Diabetes Research (DZD e.V.; 01GI0925), the project "National Institute of Mental Health (NIMH-CZ)" grant ED2.1.00/03.0078, the European Regional Development Fund, and Czech Science Foundation (GAČR grant 14-03627S). Institutional support for the Institute of Physiology, CAS was provided by RVO: 67985823.

Literature

Agarwal, G., Stevenson, I.H., Berényi, A., Mizuseki, K., Buzsáki, G., \& Sommer, F.T. (2014). Spatially distributed local fields in the hippocampus encode rat position. Science, 344, 626-630.

Bonnevie, T., Dunn, B., Fyhn, M., Hafting, T., Derdikmann, D., Kubie, J.L., Roudi, Y., Moser, E.I., \& Moser, M-B. (2013). Grid cells require excitatory drive from the hippocampus, Nat. Neurosci., 16, 309317.

Brown, J.E., \& Skaggs, W.E. (2002). Concordant and discordant coding of spatial location in populations of hippocampal CA1 pyramidal cells. J. Neurophysiol., 88, 1605-1613.

Buzsaki, G. (2010). Neural syntax: cell assemblies, synapsembles, and readers. Neuron, 68, 362-385.

Buzsaki, G., Anastassiou, C.A., \& Koch, C. (2012). The origin of extracellular fields and currents--EEG, ECoG, LFP and spikes. Nat. Rev. Neurosci., 13, 407-420.

Carr, M.F., Jadhav, S.P., \& Frank, L.M. (2011). Hippocampal replay in the awake state: a potential substrate for memory consolidation and retrieval. Nat. Neurosci., 14, 147-153.

Carter, C.S., \& Barch, D.M. (2007). Cognitive neuroscience-based approaches to measuring and improving treatment effects on cognition in schizophrenia: the CNTRICS initiative. Schizophr. Bull., 33, 1131-1137.

Eichenbaum, H., Kuperstein, M., Fagan, A., \& Naqode, J. (1987). Cue-sampling and goal-approach correlates of hippocampal unit activity in rats performing an odor-discrimination task. J. Neurosci., 7(3), 716-732. 
Fenton, A.A., Csizmadia, G., \& Muller, R.U. (2000a). Conjoint control of hippocampal place cell firing by two visual stimuli. I. The effects of moving the stimuli on firing field positions. J. Gen. Physiol., 116, 191-209.

Fenton, A.A., Csizmadia, G., \& Muller, R.U. (2000b). Conjoint control of hippocampal place cell firing by two visual stimuli. II. A vector-field theory that predicts modifications of the representation of the environment. J. Gen. Physiol., 116, 211-221.

Fenton, A.A., \& Muller, R.U. (1998). Place cell discharge is extremely variable during individual passes of the rat through the firing field. Proc. Natl. Acad. Sci. U. S. A., 95, 3182-3187.

Fenton, A.A., Kao, H-Y., Neymotin, S.A., Olypher, A., Vayntrub, Y., Lytton, W.W., \& Ludvig, N. (2008). Unmasking the CA1 ensemble place code by exposures to small and large environments: More place cells and multiple, irregularly arranged, and expanded place fields in the larger space. J. Neurosci., 28, 11250-11262.

Fenton, A.A., Lytton, W.W., Barry, J.M., Lenck-Santini, P.P., Zinyuk, L.E., Kubík, S., Bures, J., Poucet, B., Muller, R.U., \& Olypher, A.V. (2010). Attention-like modulation of hippocampus place cell discharge. J. Neurosci., 30, 4613-4625.

Fenton, A.A. (2015). Excitation-inhibition discoordination in rodent models of mental disorders. Biol. Psych. in press.

Gothard, K.M., Skaggs, W.E., Moore, K.M., \& McNaughton, B.L. (1996a). Binding of hippocampal CA1 neural activity to multiple reference frames in a landmark-based navigation task. J. Neurosci., 16, 823-835.

Gothard, K.M., Skaggs, W.E., \& McNaughton, B.L. (1996b). Dynamics of mismatch correction in the hippocampal ensemble code for space: interaction between path integration and environmental cues. J. Neurosci., 16, 8027-8040.

Gothard, K.M., Hoffman, K.L., Battaglia, F.P., \& McNaughton, B.L. (2001). Dentate gyrus and CA1 ensemble activity during spatial reference frame shifts in the presence and absence of visual input. $J$. Neurosci., 21, 7284-7292.

Gupta, A.S., van der Meer, M.A., Touretzky, D.S., \& Redish, A.D. (2010). Hippocampal replay is not a simple function of experience. Neuron, 65, 695-705.

Guzowski, J.F., Knierim, J.J., \& Moser, E.I. (2004). Ensemble dynamics of hippocampal regions CA3 and CA1. Neuron, 44, 581-584.

Hampson, R.E., Heyser, C.J., \& Deadwyler, S.A. (1993). Hippocampal cell firing correlates of delayedmatch-to-sample performance in the rat. Behav. Neurosci., 107, 715-739.

Harris, K.D., Csicsvari, J., Hirase, H., Dragoi, G., \& Buzsáki, G. (2003). Organization of the cell assemblies in the hippocampus. Nature, 424, 552-556.

Hasselmo, M.E. (2005). What is the function of hippocampal theta rhythm? - Linking behavioral data to phasic properties of field potential and unit recording data. Hippocampus, 15, 936-949. 
Hebb, D.O. (1949). The organization of behavior, A neuropsychological theory, John Wiley and Sons, New York

Ito, H.T., Zhang, S.J., Witter, M.P., Moser, E.I., \& Moser, M-B. (2015). A prefrontal-thalamohippocampal circuit for goal-directed spatial navigation. Nature, 522, 50-55.

Jackson, J. \& Redish, A.D. (2007). Network dynamics of hippocampal cell-assemblies resemble multiple spatial maps within single tasks. Hippocampus, 17, 1209-1229.

Jezek, K., Henriksen, E.J., Treves, A., Moser, E.I., \& Moser, M-B. (2011). Theta-paced flickering between place-cell maps in the hippocampus. Nature, 478, 246-249.

Johnson, A., \& Redish, A.D. (2007). Neural ensembles in CA3 transiently encode paths forward of the animal at a decision point. J. Neurosci., 27(45), 12176-12189.

Kelemen, E., \& Fenton, A.A. (2010). Dynamic grouping of hippocampal neural activity during cognitive control of two spatial frames. PLoS Biol., 8(6):e1000403. doi:

10.1371/journal.pbio.1000403.

Kelemen, E., \& Fenton, A.A. (2012). The organization of neuronal discharge on timescales of milliseconds and seconds is related to the spatial response properties of hippocampal neurons, 1 edn (New York: Springer-Verlag).

Kelemen, E., \& Fenton, A.A. (2013). Key features of human episodic recollection in the cross-episode retrieval of rat hippocampus representations of space. PLoS Biol., 11(7):e1001607. doi: 10.1371/journal.pbio.1001607.

Knierim, J.J., Kudrimoti, H.S., \& McNaughton, B.L. (1995). Place cells, head direction cells, and the learning of landmark stability. J. Neurosci., 15, 1648-1659.

Knierim, J.J. (2002). Dynamic interactions between local surface cues, distal landmarks, and intrinsic circuitry in hippocampal place cells. J. Neurosci., 22, 6254-6264.

Knierim, J.J., \& Zhang, K. (2012). Attractor dynamics of spatially correlated neural activity in the limbic system. Annu. Rev. Neurosci., 35, 267-285

Lasztoczi, B., \& Klausberger, T. (2014). Layer-Specific GABAergic Control of Distinct Gamma Oscillations in the CA1 Hippocampus. Neuron, 81, 1126-1139.

Lee, I., Yoganarasimha, D., Rao, G., \& Knierim, J.J. (2004). Comparison of population coherence of place cells in hippocampal subfields CA1 and CA3. Nature, 430, 456-459.

Lesh, T.A., Niendam, T.A., Minzenberg, M.J., \& Carter, C.S. (2011). Cognitive control deficits in schizophrenia: mechanisms and meaning. Neuropsychopharmacology, 36, 316-338.

Leutgeb, S., Leutgeb, J.K., Treves, A., Moser, M-B., \& Moser, E.I. (2004). Distinct ensemble codes in hippocampal areas CA3 and CA1. Science, 305, 1295-1298. 
Leutgeb, J.K., Leutgeb, S., Treves, A., Mayer, R., Barnes, C.A., McNaughton, B.L., Moser, M-B., \& Moser, E.I. (2005a). Progressive transformation of hippocampal neuronal representations in 'morphed' environments. Neuron, 48, 345-358.

Leutgeb, S., Leutgeb, J.K., Barnes, C.A., Moser, E.I., McNaughton, B.L., \& Moser, M.B. (2005b). Independent codes for spatial and episodic memory in hippocampal neuronal ensembles. Science, 309, 619-623.

Mankin, E.A., Sparks, F.T., Slayyeh, B., Sutherland, R.J., Leutgeb, S., \& Leutgeb, J.K. (2012). Neuronal code for extended time in the hippocampus. Proc. Natl. Acad. Sci. USA, 109, 19462-19467.

Manns, J.R., Howard, M.W., \& Eichenbaum, H. (2007). Gradual changes in hippocampal activity support remembering the order of events. Neuron, 56, 530-540.

Markus, E.J., Qin, Y.L., Leonard, B., Skaggs, W.E., McNaughton, B.L., \& Barnes, C.A. (1995). Interactions between location and task affect the spatial and directional firing of hippocampal neurons. J. Neurosci., 15, 7079-7094.

Marr, D. (1971). Simple memory: a theory for archicortex. Philos. Trans. R. Soc. Lond. B. Biol. Sci., $262,23-81$.

McNaughton, B.L., Barnes, C.A., \& O'Keefe, J. (1983). The contribution of position, direction, and velocity to single unit activity in the hippocampus of freely-moving rats. Exp. Brain Res., 52, 41-49.

Muller, R.U., \& Kubie, J.L. (1987). The effects of changes in the environment on the spatial firing of hippocampal complex-spike cells. J. Neurosci., 7, 1951-1968.

Muller, R.U., Kubie, J.L., \& Ranck, J.B. Jr. (1987). Spatial firing patterns of hippocampal complexspike cells in a fixed environment. J. Neurosci., 7, 1935-1950.

Muller, R. (1996). A quarter of a century of place cells. Neuron, 17, 813-822.

Okun, M., Steinmetz, N.A., Cossell, L., lacaruso, M.F., Ko, H., Bartho, P., Moore, T., Hofer, S.B., MrsicFlogel, T.D., Carandini, M., \& Harris, K.D. (2015). Diverse coupling of neurons to populations in sensory cortex. Nature, 521, 511-515.

Olypher, A.V., Lánský, P., \& Fenton, A.A. (2002). Properties of extra-positional signal in hippocampal place cell discharge derived from the overdispersion in location-specific firing. Neurosci., 111, 553566.

Olypher, A.V., Klement, D., \& Fenton, A.A. (2006). Cognitive disorganization in hippocampus: a physiological model of the disorganization in psychosis. J. Neurosci., 26, 158-168.

O'Keefe, J., \& Dostrovsky, J. (1971). The hippocampus as a spatial map. Preliminary evidence from unit activity in the freely-moving rat. Brain Res., 34, 171-175.

O'Reilly, K.C., Kao, H-Y., Lee, H., \& Fenton, A.A. (2014). Converging on a core cognitive deficit: the impact of various neurodevelopmental insults on cognitive control. Front. Neurosci., 8, doi: 10.3389/fnins.2014.00153 
O’Reilly, R.C., \& McClelland, J.L. (1994). Hippocampal conjunctive encoding, storage, and recall: avoiding a trade-off. Hippocampus. 4, 661-682.

Phillips, W.A., \& Silverstein, S.M. (2003). Convergence of biological and psychological perspectives on cognitive coordination in schizophrenia. Behav. Brain Sci., 26, 65-82; discussion 82-137.

Posner, M.I., \& Snyder, C.R.R. (1975). Attention and cognitive control. In Information processing and cognition: the Loyola symposium, R.L. Solso, ed. (Hillsdale, N.J: L. Erlbaum Associates).

Redish, A.D. (1999). Beyond the cognitive map: From place cells to episodic memory. Cambridge. MIT Press

Redish, A.D., Rosenzweig, E.S., Bohanick, J.D., McNaughton, B.L., \& Barnes, C.A. (2000). Dynamics of hippocampal ensemble activity realignment: time versus space. J. Neurosci., 20, 9298-9309.

Ridderinkhof, K.R., van der Wildenberg, W.P., Segalowitz, S.J., \& Carter, C.S. (2004). Neurocognitive mechanisms of cognitive control: the role of prefrontal cortex in action selection, response inhibition, performance monitoring, and reward-based learning. Brain. Cogn., 56, 129-140.

Rivard, B., Li, Y., Lenck-Santini, P.P., Poucet, B., \& Muller, R.U. (2004). Representation of objects in space by two classes of hippocampal pyramidal cells. J. Gen. Physiol., 124, 9-25.

Rosenzweig, E.S., Redish, A.D., McNaughton, B.L., \& Barnes, C.A. (2003). Hippocampal map realignment and spatial learning. Nat. Neurosci., 6, 609-615.

Royer, S., Zemelman, B.V., Losonczy, A., Kim, J., Chance, F., Magee, J.C., \& Buzsaki, G. (2012). Control of timing, rate and bursts of hippocampal place cells by dendritic and somatic inhibition. Nat. Neurosci., 15, 769-775.

Samsonovich, A., \& McNaughton, B.L. (1997). Path integration and cognitive mapping in a continuous attractor neural network model. J. Neurosci., 17, 5900-5920.

Schomburg, E.W., Fernández-Ruiz, A., Mizuseki, K., Berényi, A., Anastassiou, C.A., Koch, C., \& Buzsáki, G. (2014). Theta phase segregation of input-specific gamma patterns in entorhinal-hippocampal networks. Neuron, 84, 470-485.

Shapiro, M.L., Tanila, H., \& Eichenbaum, H. (1997). Cues that hippocampal place cells encode: dynamic and hierarchical representation of local and distal stimuli. Hippocampus, 7, 624-642.

Sharp, P.E., Blair H.T., Etkin, D., \&Tzanetos, D.B. (1995). Influence of vestibular and visual motion information on the spatial firing patterns of hippocampal place cells. J. Neurosci., 15, 173-189.

Skaggs, W.E., \& McNaughton, B.L. (1998). Spatial firing properties of hippocampal CA1 populations in an environment containing two visually identical regions. J. Neurosci., 18, 8455-8466

Tanila, H., Shapiro, M.L., \& Eichenbaum, H. (1997a). Discordance of spatial representation in ensembles of hippocampal place cells. Hippocampus, 7, 613-623.

Tanila, H., Shapiro, M., Gallagher, M., \& Eichenbaum, H. (1997b). Brain aging: changes in the nature of information coding by the hippocampus. J. Neurosci., 17, 5155-5166. 
Uhlhaas, P.J., \& Singer, W. (2006). Neural synchrony in brain disorders: relevance for cognitive dysfunctions and pathophysiology. Neuron, 52, 155-168.

Uhlhaas, P.J., \& Singer, W. (2007). What do disturbances in neural synchrony tell us about autism? Biol. Psychiatry., 62, 190-191.

Vazdarjanova, A., \& Guzowski, J.F. (2004). Differences in hippocampal neuronal population responses to modifications of an environmental context: Evidence for distinct, yet complementary, functions of CA3 and CA1 ensembles. J. Neurosci., 24, 6489-6496.

Wesierska, M., Dockery, C., \& Fenton, A.A. (2005). Beyond memory, navigation, and inhibition: behavioral evidence for hippocampus-dependent cognitive coordination in the rat. J. Neurosci., 25, 2413-2419.

Wills, T.J., Lever, C., Cacucci, F., Burgess, N., \& O'Keefe, J. (2005). Attractor dynamics in the hippocampal representation of the local environment. Science. 308, 873-876.

Wilson, I.A., Ikonen, S., Gallagher, M., Eichenbaum, H., \& Tanila, H. (2005). Age-associated alternations of hippocampal place cells are subregion specific. J. Neurosci., 25, 6877-6886.

Wood, E.R., Dudchenko, P.A., Robitsek, R.J., \& Eichenbaum, H. (2000). Hippocampal neurons encode information about different types of memory episodes occurring in the same location. Neuron, 27, 623-633.

Ziv, Y., Burns, L.D., Cocker, E.D., Hamel, E.O., Ghosh, K.K., Kitch, L.J., El Gamal, A., \& Schnitzer, M.J. (2013). Long-term dynamics of CA1 hippocampal place codes. Nat. Neurosci., 16, 264-266. 
A $\quad B$

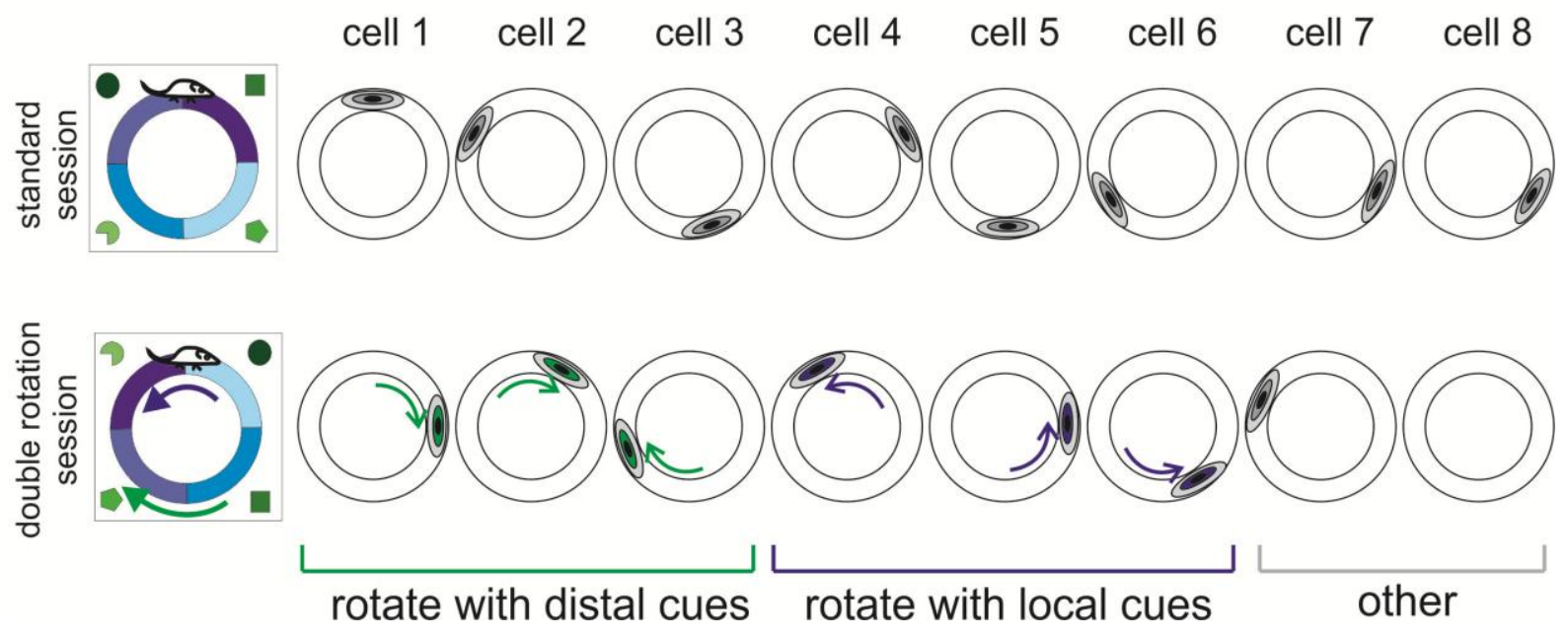

Figure 1. Hippocampal CA1 cells represent multiple sets of landmarks within a single session (cartoon based on fig. 2 of Knierim, 2002). A) Design of the experiment: An ensemble of CA1 place cells was recorded as a rat ran on a circular track with a set of intra-maze (local) cues (shown in blue) and a set of extra-maze (distal) cues (shown in green). The same ensemble of cells was recorded in two sessions: In a standard session, and in a double rotation session, in which the local cues were rotated by a fixed amount $\left(90^{\circ}\right.$ in this example) in one direction (counter clock-wise) and the distal cues were rotated in the opposite direction $\left(90^{\circ}\right.$ clock-wise) to dissociate the local and distal spatial frames of locations. B) Responses of an ensemble of simultaneously recorded place cells to the manipulation shown in panel $A$. The top row shows the locations where cells discharged on the track during a standard session and the bottom row shows the corresponding discharge of each cell during a double rotation session. The firing fields of cells 1,2 and 3 followed distal cues. The firing of cells 4 , 5 and 6 followed local cues, and the remaining two cells changed their firing independently of the two sets of cues. 

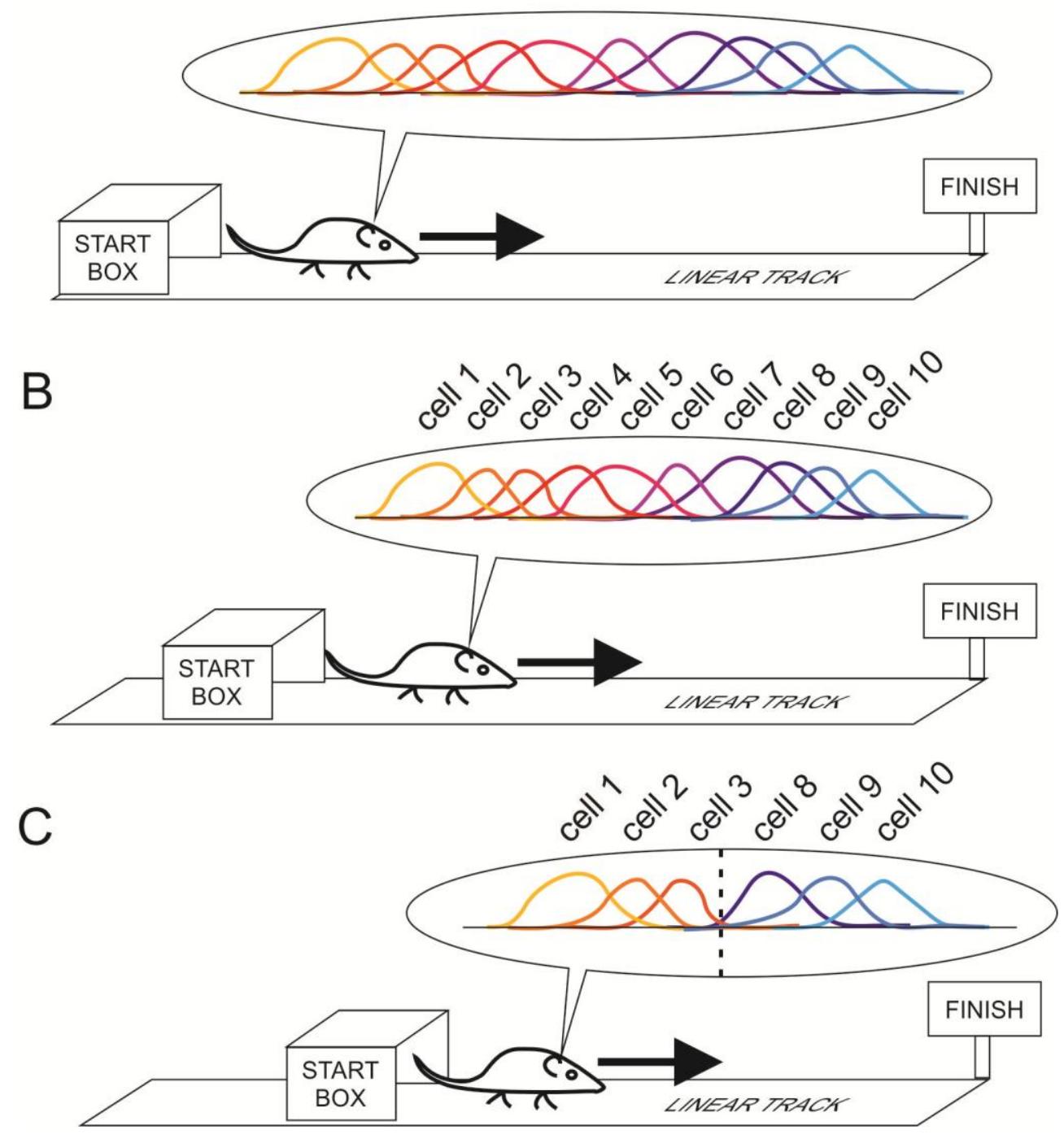

Figure 2. Transition between two CA1 ensemble representations within a session (cartoon based on fig. 4 and 7 of Gothard et al., 1996b). Ensembles of CA1 place cells were recorded as a rat walked between two endpoints of a linear track, one of which was equipped with a box. On different trials the position of the box changed relative to the track ends and room landmarks, creating journeys of different length $(A, B$, and $C$ ). Examples show responses of 10 CA1 place cells during journeys with different location of the box. The 'hill' shows for each place cell an area of increased activity along the track. At the beginning of journeys, the neural activity was aligned with the spatial frame defined by the box, but at the end of the journeys it was aligned with the spatial frame of the room. During the short journeys the transition between box-bound representations of position at the beginning of journeys, and track-bound representations of position at the end of the journeys could be gradual (B) or abrupt (C). While time averaged data suggest a gradual transition (B), Redish et al. (2000) analysed single journeys of the rat and observed abrupt transitions between representations like in panel (C). 
A

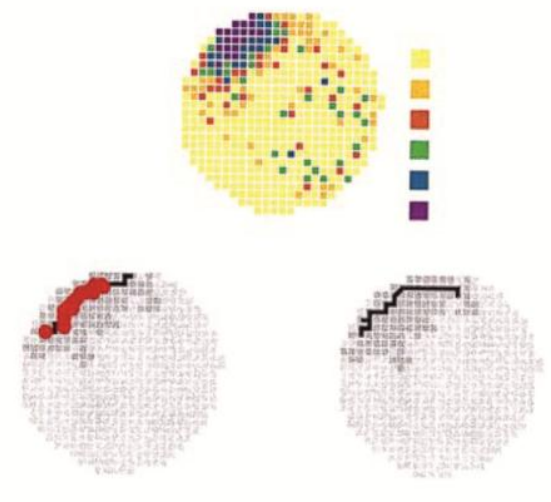

B

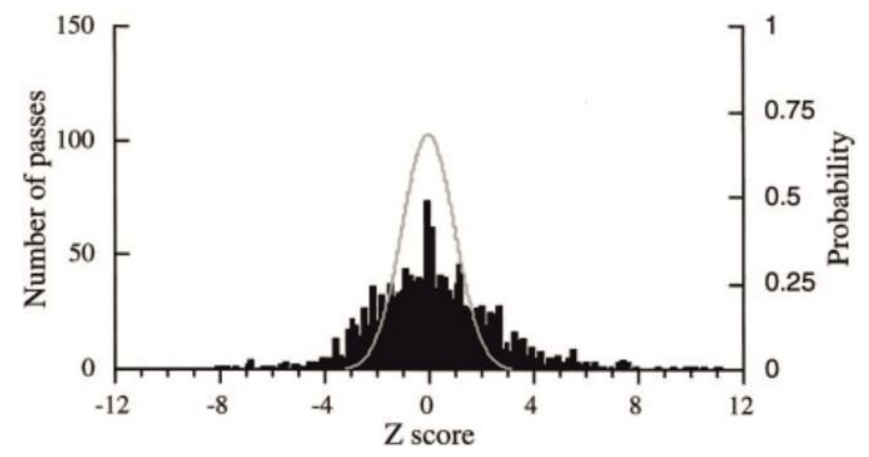

Figure 3. Overdispersion in place cell firing (reproduced with permission from Fenton and Muller, 1998). A) An example illustrating the variability of firing of a single place cell during similar passes of a rat through the firing field in the same recording session. At the top is a firing rate map of a cell recorded as a rat foraged in a cylinder. Darker colors indicate locations with higher firing rate. The bottom pair of maps illustrates discharge of the cell during two passes through the firing field. The rat's path is black and the locations of spikes are red. Although the two paths are very similar, the cell fired 18 spikes during the path at the left, but no spikes on the path at the right. B) The variability of firing was quantified by converting the firing rate during each pass of the rat through the firing field to a $z$ score. The distribution of these $z$ scores (shown in black) was compared to the distribution expected of an inhomogeneous Poisson model (gray line). The comparison reveals that the variability in place cell firing is higher than expected by chance. 
A

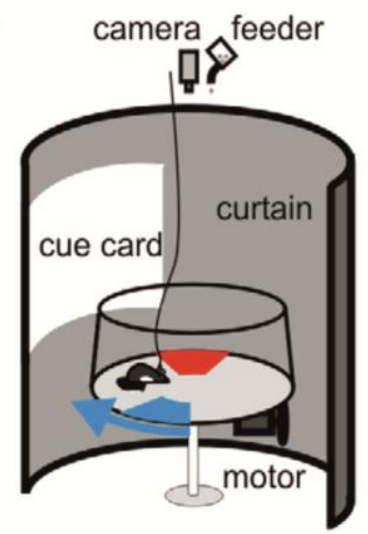

B

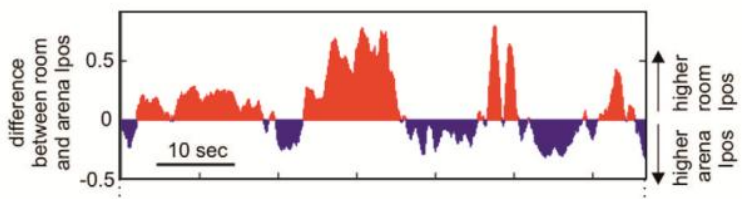

C

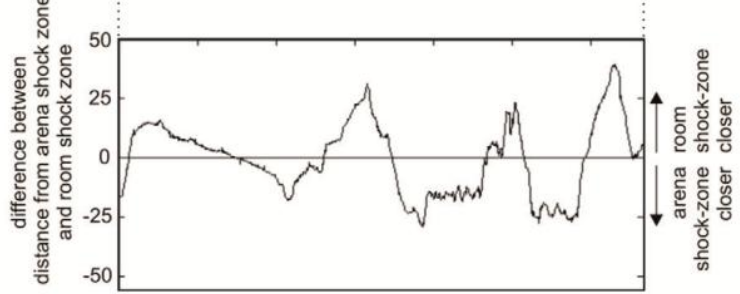

Figure 4. Dynamic functional grouping of representations of positions in two spatial frames and its relationship to ongoing behavior (reproduced with permission from Kelemen and Fenton, 2010). A) In a two-frame, active place avoidance task, a rat on a slowly rotating arena ( $1 \mathrm{rpm}$ ) was reinforced to multitask: to avoid a room shock zone (red) defined relative to stable room cues, and to avoid an arena shock zone (blue) defined relative to rotating arena cues. B) The momentary positional information computed from the ensemble firing of CA1 cells alternated between periods of predominant arena information (blue), and periods of predominant room information (red). C) The difference between the rat's distance to the arena shock zone and distance to the room shock zone during the same time interval as in $\mathrm{B}$. The information about position that is predominantly present in CA1 discharge tracks the relative distance of the rat to the two shock zones, with room information prevailing when the rat was closer to the room shock zone and vice versa. At a particular moment the more behaviorally relevant information tends to be better represented in the ensemble discharge of CA1 cells. 

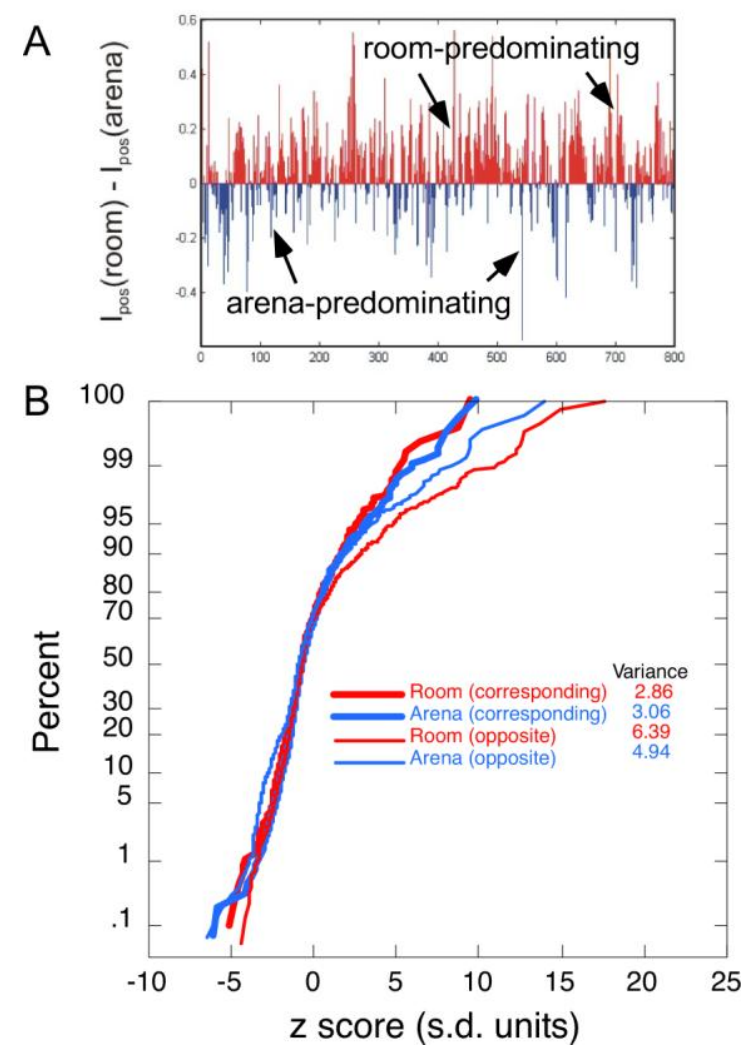

Figure 5. Overdispersion during the multitasking behavior in which the dynamic functional grouping of place cell ensembles was identified. A) The recordings were subdivided into the episodes when room information predominated in the CA1 ensemble (room episodes), and the moments when arena information predominated (arena episodes). B) Cumulative probability distribution of $\mathrm{z}$ scores for the room spatial frame during room episodes (var = 2.86); for arena spatial frame during arena episodes (var = 3.06); for room spatial frame during arena episodes (var =6.39); and for the arena spatial frame during room episodes (var $=4.94)$. 


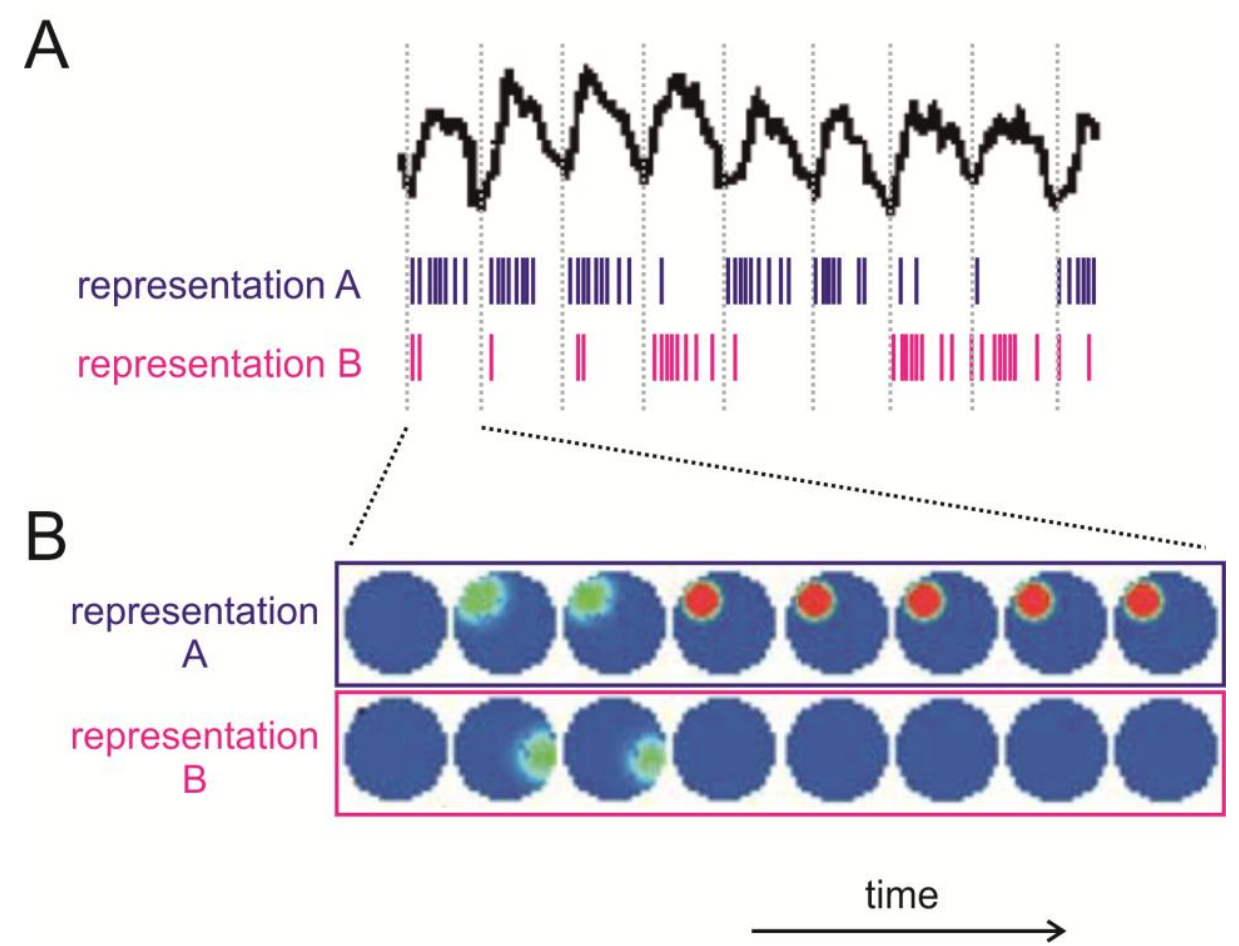

Figure 6. Switching of representations organized by theta oscillations in the local field potential. A) Within each theta cycle one of the competing potentially relevant representations (representation $A$ - purple, and representation B - magenta) dominates hippocampal activity. The raster plots indicate pyramidal cell activity related to the two representations. B) At the phase of the theta cycle when hippocampal activity is less inhibited, different representations may compete in a winner-take-all manner, such that later in the cycle when inhibition is greater, the activity pattern settles into an attractor state that corresponds to a single representation. 

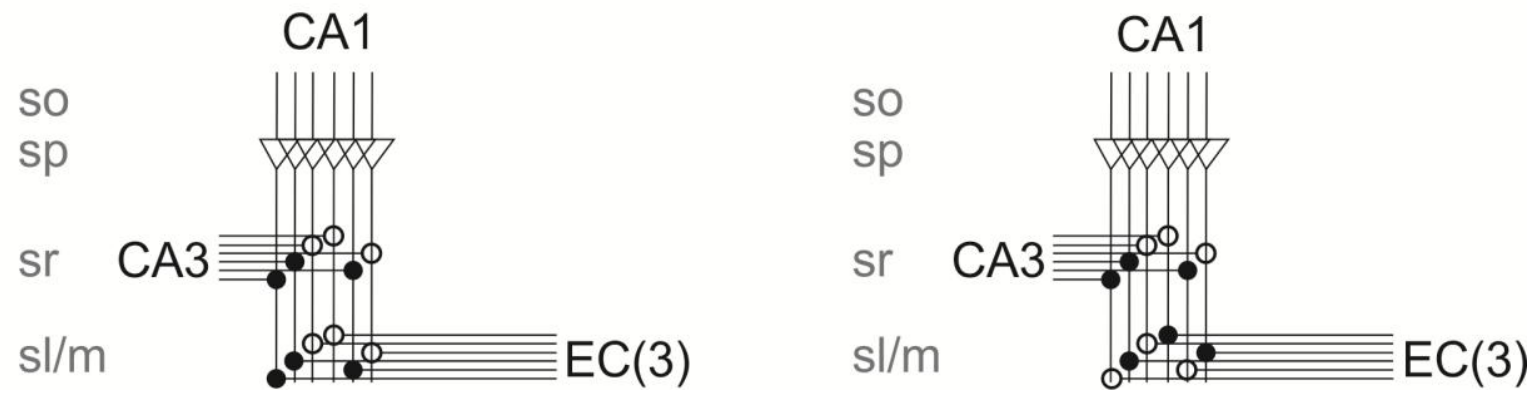

Figure 7. Hippocampus CA1 is in position to detect the (mis-) match between the representation that is retrieved from memory (arriving from CA3 region) and less-processed information from entorhinal cortex. A) Depiction of a match situation when information-consistent inputs from CA3 (via the Schaffer collaterals) and the entorhinal cortex (via the perforant path) converge on the same neurons. B) Depiction of a mismatch case when the synaptic inputs from CA3 and the entorhinal cortex arrive on different CA1 neurons. (so - stratum oriens, $\mathrm{sp}$ - stratum pyramidale, sr - stratum radiatum, sl/m - stratum lacunosum moleculare) 